\title{
Chapter 8 \\ The Jet: Fluid in Motion and More Vorticity
}

A jet is always involved in propelling any object through a fluid medium, even by living creatures. A propeller creates a jet and does it mechanically. The so-called jet engine does it by other means that are described later in our story. At this level of looking at the physics, there is no difference between piston engine driven propellers and a jet engine: both create jets of air.

The discussion of wings lifting an airplane noted that a vortex must form a closed loop or end on a boundary. A beautiful example of a boundary is a water surface under which we can create a jet with a paddle and observe the ends of a vortex loop (Fig. 8.1). Under the water is a "U" shaped vortex that is akin to the smoke ring blown by the person in Fig. 6.3. In the flight environment, such boundaries are sometimes harder to discern if they exist at all.

As we stand next to a person blowing the smoke ring (Fig. 6.3), the ring will be seen to proceed in the blown direction at a modest speed. That speed has to do with the strength of the vortex and its size. Specifically, the velocity induced by the vortex from the portion at 3 o'clock interacts with the portion at the 9 o'clock position and that interaction leads to force just like the bound vortex on the wing provides lift. Now imagine the person can blow many rings in rapid succession so that the vortices form a tube. We can describe a vortex tube as a jet boundary. Returning to the canoe paddle example, the jet can be visualized there if the paddler strokes the water in rapid succession. He will leave an aligned array of vortex pairs as evidence of the jet he created. This is precisely how a stern-wheeler ship is propelled. Taking the water example a step further, we note that a fish leaves alternating vortices at his left and right rear when it sweeps its tail from side to side. Such an array is called a vortex street forming a jet to the rear and one could say that the fish is jet propelled.

The vortex pattern from a closely coupled set of rings will resemble the pattern issuing from the tip of a propeller turning at high speed. The pattern from the propeller will be helical in shape and issuing from near the blade tip. With a large number of blades, the vortex approximates a cylindrical sheet produced by the propeller. 


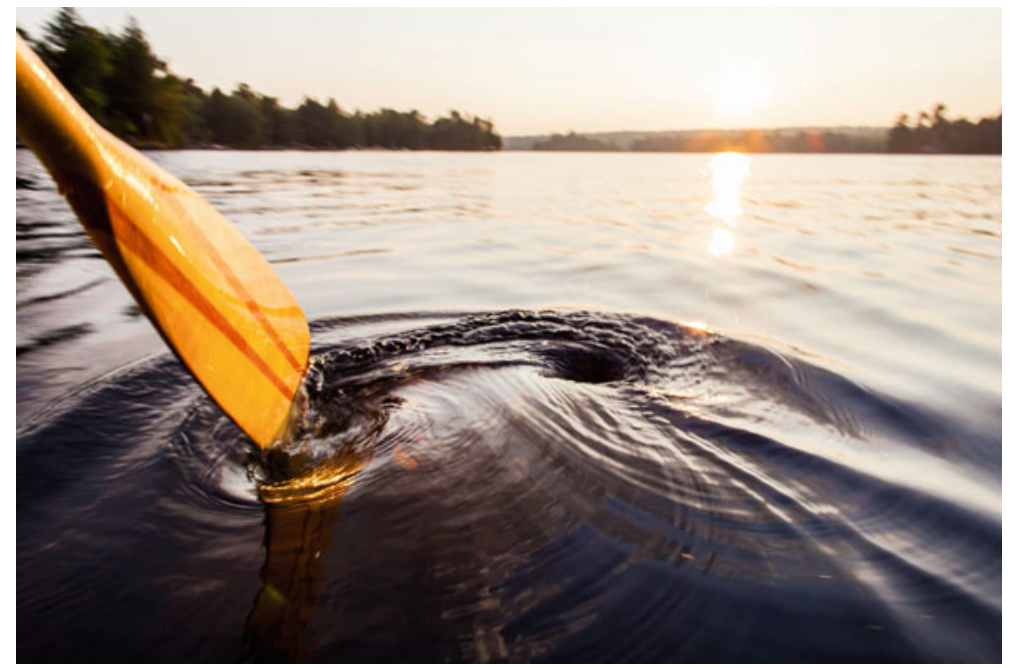

Fig. 8.1 Canoe paddle in water. Note the surface deflection associated with the locally higher rotational speeds near the center(s) (@ JP Danko/Stocksy United)

\subsection{Prop Tip Vortices}

Let us look at how vortices are involved in the creation of a jet by a propeller. The propeller blade is like a wing that rotates around a hub. As in the case of a wing, there must be a bound vortex associated with the blade and a trailing vortex from the tip.

One might ask: 'where is the closed loop of the vortex on the hub side?' A vortex can end only on a boundary and the hub may just be such a boundary, albeit not as nicely defined as a wall or surface. A more realistic description of the flow near the hub is that the vorticity trailing from the propeller blade near its hub is shed just like that from the tip. Figure 8.2 shows the vortex pattern around a single propeller blade with its root that is exaggeratedly further out from the hub than a realistic blade would be. Because the trailing vortices are helical in shape, the induced velocities have axial (the jet to the rear) and azimuthal (in the plane of propeller disc) components. Thus, both hub and tip vortices contribute to the rotating part of the jet flow. The hub vortex is not readily observable as a bundled vortex like that from a wing tip or the propeller blade tip. The reason is that the turbulence allows for a ready sharing of the vorticity with the whole of the jet. In practice, the body of the airplane or nacelle of a tractor (as opposed to a pusher) propeller interferes with an organized survival of the vorticity shed at the blade hub.

The pattern shown in Fig. 8.2 would naturally be compounded by other blades of the propeller. While the vortex issuing near the hub would likely degenerate into less organized vorticity, the condensed moisture in the tip vortex of propeller can, under certain conditions, remain organized and visible as seen in Fig. 8.3. 


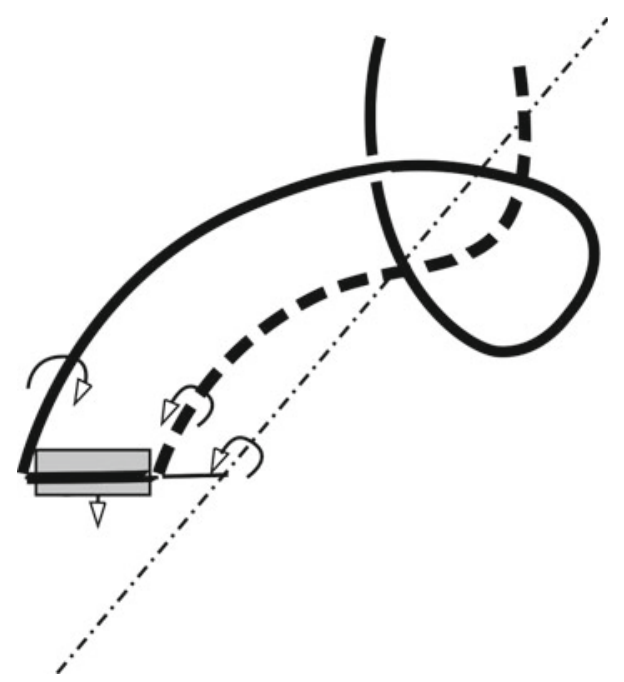

Fig. 8.2 The vortex pattern from an isolated propeller blade rotating about a centerline. The trailing vortices are helical due to the blade and air motion. The blade with an exaggeratedly large hub radius is moving toward the reader
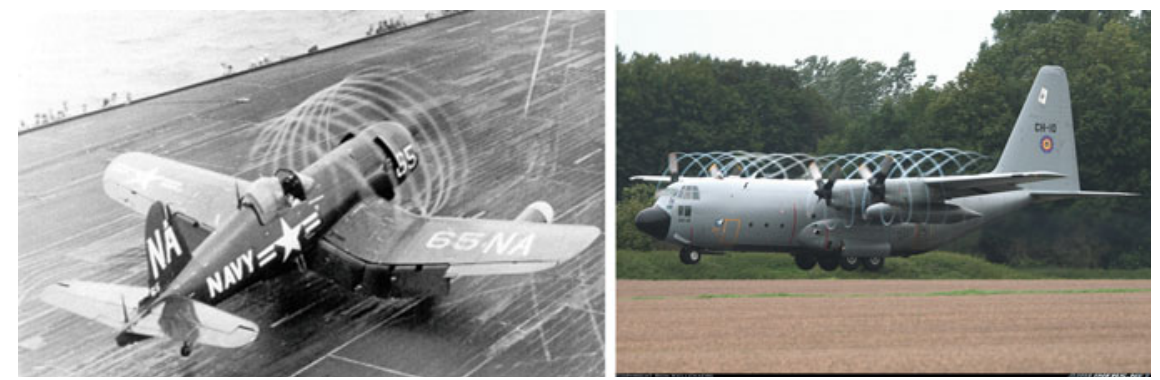

Fig. 8.3 Two examples of tip vortices made visible by atmospheric conditions. At standstill the helix is tight and expands in forward flight as shown at right (Photo at right Belgian Air Force C-130H by Ron Kellenaers \# 1131694, Airliners.net)

In contrast, the visible remainder of a turbofan jet engine in flight at altitude is a contrail. Its origin is, not from vorticity as such but, from the interaction of moisture laden engine exhaust mixing with the very cold atmospheric air at high altitudes. This is the case when there is a significant gap between the engine and the beginning of a contrail as shown, for example in Fig. 8.4. 


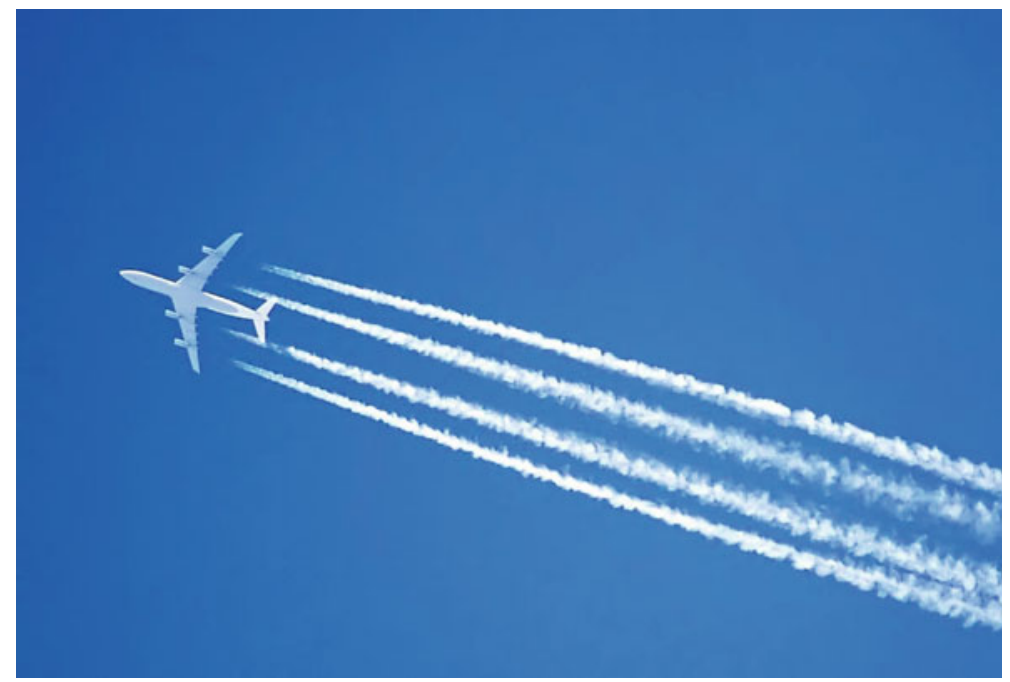

Fig. 8.4 High altitude contrails from a A340 commercial jet airliner (Photo: Adrian Pingstone; curid $=1856927$, http://commons.wikimedia.org/wiki/User:Arpingstone)

\subsection{Jet Boundaries Are Cylindrical Sheets of Vorticity}

Vorticity in a variety of forms plays a necessary role in propelling an airplane. From an overall performance viewpoint, the propeller's jet and that from a jet engine differ mainly in the amount of air processed and the speed of the jet.

In practice, the lift distribution arising from the rotating propeller blade will produce a somewhat non-uniform jet velocity because the lift distribution varies along the blade. However, the vorticity quickly assembles into a bundled vortex as the illustrations above show and the vortex sheet in the form of a tube eventually allows the jet internal velocity profile to be relatively uniform. The word 'sheet' may be described as the approximation of a sheet made of helical vortices from the ends of the blade. The jet from a jet engine tends to be uniform from the start because it exits a reservoir of pressurized gas by means of a nozzle with little rotation by virtue of the design of the turbine from which the propelling gas exits.

Unlike the wing that cruises into vertically still air and imparts downward momentum, the propulsion system must deal with the momentum associated with flight speed and direction. It must therefore manage the energy of the incoming airflow efficiently so that the installed heat engine will not have to make up for unnecessary losses. This requirement is more severe at higher flight speeds.

How does the propulsion system do that? In the case of a propeller, the blades deal with a combination of speeds: the rotational speed of the blade and the forward speed of the airplane. These two speed vectors determine the angle of incidence on the blade. Because rotational speed varies with location along the blade (faster further out), the incidence angle varies along the blade. The propeller blade is designed with twist to 
achieve a reasonably good distribution of the local angle of attack all along the blade. For airplanes that don't fly too fast, like modest cost general-aviation aircraft that may be limited to speed between 60 and $150 \mathrm{mph}(27-67 \mathrm{~m} / \mathrm{s})$, a fixed twisted blade may be adequate. This is called a fixed pitch propeller (see, for example, Fig. 2.1).

For propeller airplanes that fly faster, a mechanism is included in the design of the blade that allows the blade as a whole to be rotated about its axis at the hub. This allows optimization of the blade orientation to be realized either by mechanical adjustment by the pilot or automatically by a mechanism that senses the appropriate speeds. The historical development of propellers took place from the inception of aviation to the 1940s with ever more complex pitch-control mechanisms for ever better performance.

\subsection{An Aircraft Can Never Have 100\% Propulsive Efficiency}

... and, for the same reason, neither can a power boat! But your car can!

The key to success of the propeller as a thrust generation device for an airplane was that the speed increase in the 'jet' over the incoming freestream was low enough so that it was quite efficient. In other words, the kinetic energy investment by the propeller in the jet was modest. This notion underlies the idea that there is an efficiency that can be defined in connection with the creation of a jet by a flight vehicle propulsion system. The system is designed to create an increase in momentum, but the reality is that it produces power in the form of jet kinetic energy. Momentum depends on the velocity directly while kinetic energy depends on the square of velocity. That algebraic distinction allows stating the power delivered to the airplane relative to the power that has to be expended in order to realize the desired momentum increase. That fraction is called the propulsive efficiency of the system and is possibly the most important aspect of the system's utility because it is an important component of fuel usage. Without going through the algebraic steps, the propulsive efficiency is given by $2 /\left(1+V_{j} / V_{0}\right)$. The two symbols stand for the jet and flight velocities respectively. To express that in percent, multiply that fraction by 100 !

\subsubsection{A Quantitative Aside}

The next two paragraphs describe the origin of this critical relationship for the interested reader. The laws of mechanics state that work is done by a force moving over a distance and power is work per unit time. Thus, the power exerted by an airplane propulsion system in steady level flight is the thrust times the flight speed. The thrust is the momentum increase exerted on the stream tube that enters the propulsion system. The initial momentum is air 
mass flow rate ( $m$ in lbs/s or $\mathrm{kg} / \mathrm{s}$ ) times the flight velocity. Thus, the thrust ${ }^{1}$ is

$$
m\left(V_{j}-V_{0}\right) \text { and the power exerted is } m\left(V_{j}-V_{0}\right) \cdot V_{0}
$$
the jet:

The power provided by the propulsion system is the net mechanical power in the air of

$$
1 / 2 m\left(V_{j}^{2}-V_{0}^{2}\right)
$$

The ratio of these two power quantities gives the propulsive efficiency noted above.

We note here that the mass flow rate $(m)$ is all the air processed by the propulsion system. For a fan jet, that includes both the fan flow and the primary or 'core' flow through the components of the engine proper. In that sense, the velocity of the jet is an average that may or may not be mixed in an actual turbofan engine.

One hundred percent efficiency is realized when the two velocities are the same. These two velocities are identical if you look at the wheel of your car being the propulsion system and the almost infinite Earth is the medium. That also means that the momentum increase of the medium is close to zero meaning that by moving our car, no energy is left in the medium. This silly example is an accurate description of a wheel (a propulsion system) in contact with the ground where we enjoy one hundred percent propulsive efficiency, provided we don't spin wheels and thereby shoot gravel at a neighbor's window.

Typical propulsive efficiencies for propeller driven airplanes range around eighty percent and better, depending on the sophistication of the geometric design and operation of the propeller. Arguments were made against the practicality of the jet engine because the exhaust velocity could be calculated and determined to be quite high. For a nominal and realistic (at the time) speed ratio (jet to flight) of 3 to 1 , the efficiency is just $50 \%$, judged to lead to an excessive fuel consumption rate by the engine. That argument was ultimately judged to be false (or at least could be overcome) and proponents did manage to build what we call a jet engine.

\subsection{Counter-Rotating Props}

In addition to this aspect of efficiency, the conventional propeller imparts a rotation to the air processed by it by virtue of the drag forces similar to those on a wing: pressure, viscous and induced drag components. We have suggested in the earlier text that the induced drag is responsible for the rotation of a propeller's jet. Such rotation represents rotational kinetic energy in the 'jet' stream behind a propeller. If nothing else is done, this energy is wasted and presents an additional loss that,

\footnotetext{
${ }^{1}$ For an accurate statement regarding engine thrust, there may be a pressure term in addition to the momentum term. In air-breathing propulsion this term is usually small and neglected here. Not so in connection with rocket engines discussed in Chap. 13.
} 

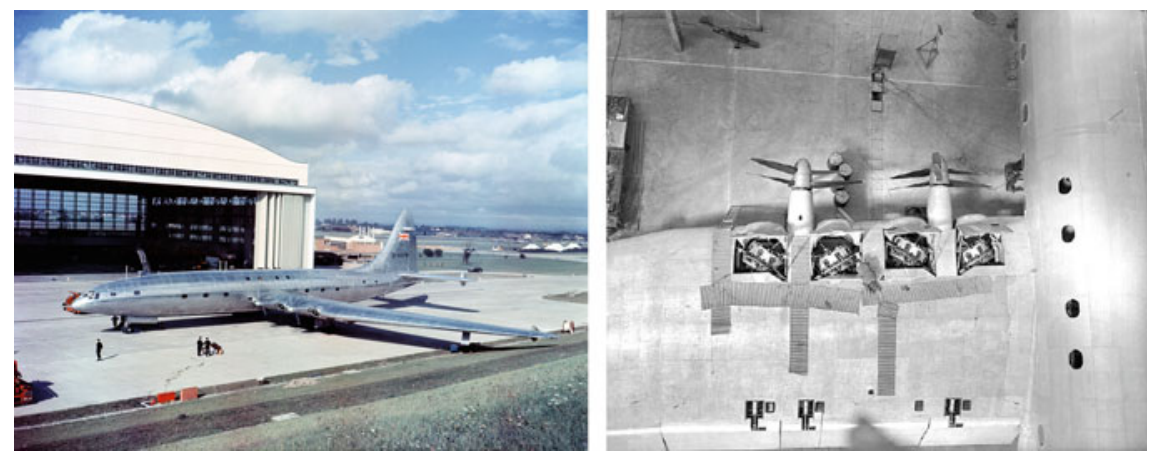

Fig. 8.5 The British Bristol Brabazon airliner prototype and its piston engine installation for counter-rotating propellers (Photos courtesy BAE SYSTEMS)

if sufficient in magnitude, might have to be dealt with. It contributes nothing to the propeller thrust and is eventually dissipated in the turbulence of the jet.

In a number of aircraft with propeller engine configurations, the loading of the propeller was sufficiently high that something had to be done to recover the energy in the rotation of the 'jet' stream. In the waning days of piston engine technology for large commercial or military airplanes, the possibility and desire arose for ever more powerful engines to drive propellers. These promised faster and larger airplanes, but they also pointed the designers to the need for fuel use efficiency to achieve long range. The wasted rotational kinetic energy had to be recovered.

In order to put more power into the 'jet' and realizing reasonable fuel usage while keeping propeller diameters reasonable, airplane builders turned to counter-rotating propellers. A second set of blades is tailored to take the rotating output from the first and add power in such a way as to leave little residual kinetic energy in the jet's rotation motion. The nature of the jet flow behind a propeller is not nice and clean, so that to placing two propeller sets in tandem in this way is challenging to do well, but it is somewhat effective. Examples of such configurations are the British Bristol Brabazon (Fig. 8.5) and the Tupolev Tu-95 strategic bomber built in the Soviet Union during the Cold War shown Fig. 8.6. The Brabazon airliner used two piston engines $^{2}$ to drive each propeller set while the Tu-95 used a single gas turbine engine. In both cases, four set of two propellers each were used, two on each wing, eight engines in total. Gearing was necessarily required. The concept never went very far for economic reasons and gearing always comes with weight, power consumption, and reliability issues of its own. Further, an airliner conversion of the Tupolev bomber proved the concept to be very noisy for passengers. The turbojet age was dawning and was about to change history. In the US, the counter-rotating propeller concept was never brought to a widespread reality. One place where it was considered was for a number of vertical takeoff military fighter prototypes (Fig. 8.7). For such airplanes

\footnotetext{
${ }^{2}$ The eight power plants for this airplane were Bristol Centaurus engines that sported sleeve-valves.
} 


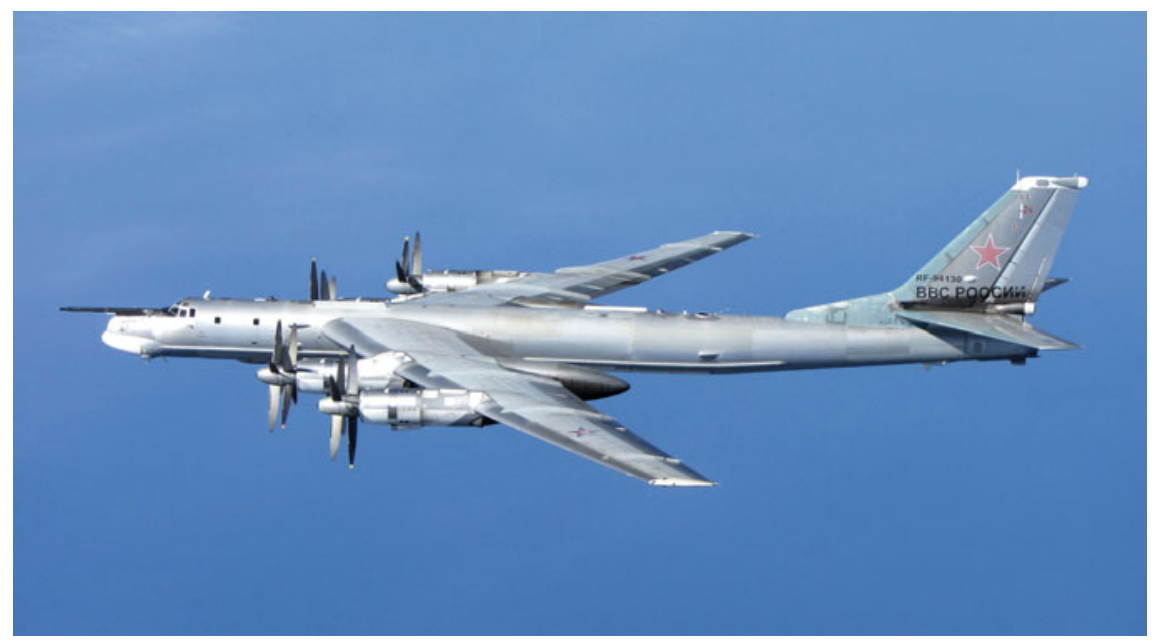

Fig. 8.6 The Cold War Soviet Tupolev Tu-95 strategic bomber using counter-rotating propellers

Fig. 8.7 Convair XFY-1 "Pogo" prototype, a vertical takeoff and landing fighter (1954) with counter-rotating propellers powered by a turboprop engine. A similar Lockheed airplane was built for a competition that did not lead to a production contract (US Navy)

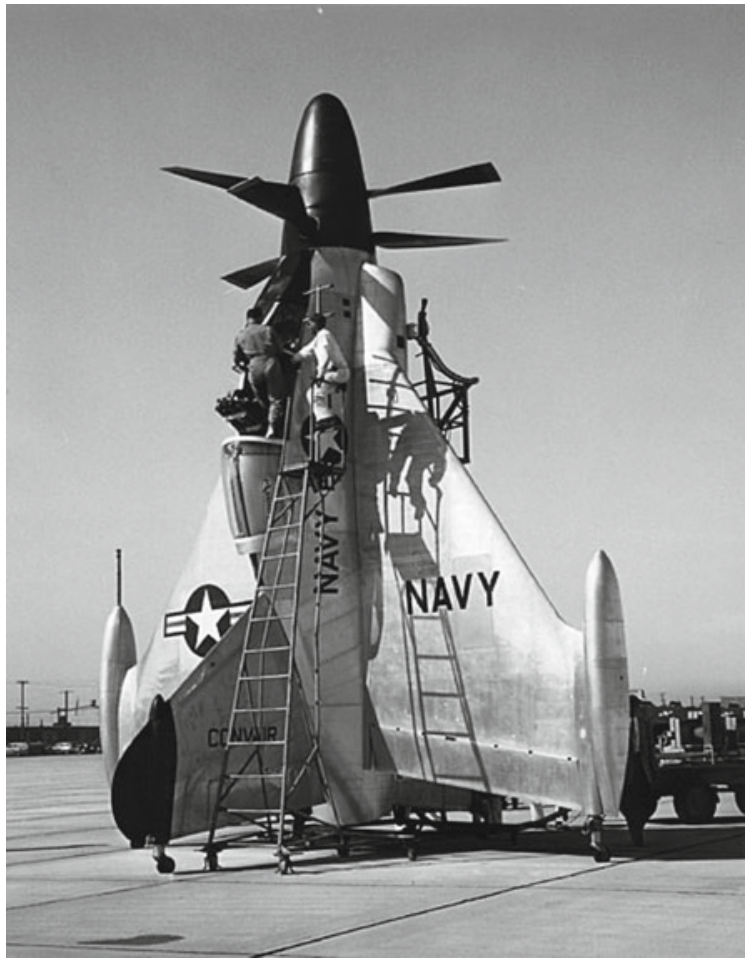


to leave residual rotating flow in the lifting jet means that the airplane will undergo a rotation as a reaction. This may present an undesirable control issue.

The idea of counter-rotating blades is not singular in propeller-like applications. The gas turbine will be explored in detail further on, but this is a good place to mention that the idea of counter-rotation arose in connection with gas turbine engine and its compressor. For example, during the earliest days of gas turbine engine developments in Germany (late 1930s), the thought was explored that, for a lighter weight and shorter compressor, it could have a counter-rotating set of blades rather than a rotorstator combination. It was deemed to be too complex and abandoned. Another stab at this idea was undertaken in the US in the 1970s when government contracts were let to explore the feasibility of a cowl-less turbofan for commercial airliners. This engine type resembled a counter-rotating propeller and was built around the gas turbine engines of the day. Major manufacturers tried the idea. They were called 'unducted fans' or 'propfans' and were considered for proposed airliners. These would have been constrained to flight Mach numbers around 0.7 because of the fan tip speed limitation. They found no application and the effort was deemed to be a dead-end, at the time.

The reader might wish to examine archival information concerning an attempt to operate a propeller at supersonic speeds. This is possible given sufficient power. Such a propeller was incorporated in a military airplane designated as the XF-84F. It turned out to be a spectacular failure and is said to have been the loudest airplane that ever flew!

Open Access This chapter is licensed under the terms of the Creative Commons Attribution 4.0 International License (http://creativecommons.org/licenses/by/4.0/), which permits use, sharing, adaptation, distribution and reproduction in any medium or format, as long as you give appropriate credit to the original author(s) and the source, provide a link to the Creative Commons license and indicate if changes were made.

The images or other third party material in this chapter are included in the chapter's Creative Commons license, unless indicated otherwise in a credit line to the material. If material is not included in the chapter's Creative Commons license and your intended use is not permitted by statutory regulation or exceeds the permitted use, you will need to obtain permission directly from the copyright holder.

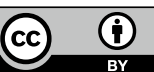

